Published in final edited form as:

J Phys Act Health. 2012 July ; 9(5): 698-705.

\title{
Validity of the Global Physical Activity Questionnaire (GPAQ) in Adult Latinas
}

\author{
Tracy Hoos, \\ Dept of Public Health, San Diego State University, San Diego, CA. \\ Nancy Espinoza, \\ Dept of Public Health, San Diego State University, San Diego, CA. \\ Simon Marshall, and \\ Dept of Exercise and Nutritional Sciences, San Diego State University, San Diego, CA. \\ Elva M. Arredondo \\ Dept of Public Health, San Diego State University, San Diego, CA.
}

\begin{abstract}
Background-Valid and reliable self-report measures of physical activity (PA) are needed to evaluate the impact of interventions aimed at increasing the levels of PA. However, few valid measures for assessing PA in Latino populations exist.
\end{abstract}

Objective-The purpose of this study is to determine whether the GPAQ is a valid measure of PA among Latinas and to examine its sensitivity to intervention change. Intervention attendance was also examined.

Methods-Baseline and postintervention data were collected from 72 Latinas (mean age $=43.01$; $\mathrm{SD}=9.05$ ) who participated in Caminando con Fe/Walking with Faith, a multilevel intervention promoting PA among church-going Latinas. Participants completed the GPAQ and were asked to wear the accelerometer for 7 consecutive days at baseline and again 6 months later. Accelerometer data were aggregated into 5 levels of activity intensity (sedentary, light, moderate, moderatevigorous, and vigorous) and correlated to self-reported mean minutes of PA across several domains (leisure time, work, commute and household chores).

Results-There were significant correlations at postintervention between self-reported minutes per week of vigorous LTPA and accelerometer measured vigorous PA $(r=.404, P<.001)$ as well as significant correlations of sensitivity to intervention change (post intervention minus baseline) between self-reported vigorous LTPA and accelerometer-measured vigorous PA $(r=.383, P<$. $003)$ and self-reported total vigorous PA and accelerometer measured vigorous PA $(r=.363, P<$. 003).

Conclusions-The findings from this study suggest that the GPAQ may be useful for evaluating the effectiveness of programs aimed at increasing vigorous levels of PA among Latinas.

\section{Keywords}

accelerometry; measurement; community; health; Hispanic

Obesity affects U.S. Latinas at disproportionate rates. The situation is particularly important within the United States Latino population. Nearly 2 out of every 3 Latinas are overweight or obese and almost $60 \%$ of Latinas are physically inactive. ${ }^{1}$ And while research suggests that physical activity (PA) habits play an important role in Latino overweight and obesity trends there is very little research validating the tools used to measure PA among this particular population. ${ }^{2}$ This is important as Latina women, in particular, have consistently 
reported lower rates of leisure time PA than non-Latina Caucasians. ${ }^{3}$ Thus, it is imperative to design interventions that will increase PA rates in this population, especially with recent reported inactivity levels as high as $60 \% .{ }^{4}$ Given that Latinas make up $48 \%$ of the estimated 30.1 million Latinos in the United States, ${ }^{5}$ investigations of PA habits of the U.S. Latina community merits further attention.

There are a number of ways to measure PA; some methods are more accurate in assessing activity than others. Accelerometry-based monitors are often used by researchers as an objective measure of PA and are generally considered the gold standard for assessing free living activity. ${ }^{5-7}$ However, accelerometers are not always feasible because they are expensive and participants have to remember to wear them during all waking hours for up to 7 days. Self-report surveys are also commonly used to assess $\mathrm{PA}^{8-10}$ but data are plagued by memory recall errors such as omission, over-reporting, and telescoping. ${ }^{11}$ In addition, PA questionnaires have not traditionally included questions that span multiple domains common among the Latina population, such as commuting, household, and work. Few self-report measures have been validated against accelerometers and even fewer self-report measures of PA have been validated specifically for use with Latinas. ${ }^{5,12,13}$ Lastly, there is a lack of evidence, especially among the Latino population, investigating whether intervention attendance increases accuracy of self-reported minutes of PA. If intervention attendance is significantly correlated to self-report accuracy, this could inform in the accuracy of evaluations of programs relying on self-report measures. Therefore, the primary objectives of this study are to 1) evaluate the validity of the GPAQ as a self-report measure among Latinas, 2) examine the GPAQ's sensitivity to intervention changes, and 3) assess whether regular intervention attendance contributes to increased accuracy of self-report data.

\section{Design}

The current study is pre and post group design. The current analysis used baseline data and postintervention data from Caminando con Fe/Walking with Faith, a year- long multilevel intervention promoting PA among churchgoing Latina women ages 18 to $65 \mathrm{yr}$. There were 97 participants in the pilot study, and 72 were adherent to the accelerometer protocol of 4 days. A sample of size of 88 is required to detect an effect size of $r=.6$, with $80 \%$ power. Thus, with a sample size of 97 , we have adequate power in a validity context. The primary dependent variable was PA at baseline and 6 months assessed through the accelerometer. Significant intervention effects were found but these data are being reported in a paper elsewhere.

\section{Participant Recruitment and Characteristics}

The selected church has 1800 enrolled parishioners (with $48 \%$ Latino congregation) and offers 2 weekly Spanish mass services. The church is located in the city of Chula Vista, California, 6 miles north from the San Diego/Baja border. Resident population is predominantly Latino, accounting for $50 \%$ of Chula Vista's population. ${ }^{14}$ To be eligible to participate, the church had to have at least 1 Spanish speaking mass service, have no current PA program, and had to be willing to participate in the program activity for a year. Church leadership was asked to identify community leaders (promotoras) who may be interested in being involved in the program. Following recommendations, the program selected 6 promotoras (3 paid, 3 volunteer) to deliver the multilevel intervention.

Latinas between the ages of 18 to 65 were recruited to participate in this study. Latinas were recruited, through flyers, church newsletters, mass announcements, and word of mouth from parish members. Of these 168 women who expressed interest in participating, 97 (58\%) Latina women were eligible and agreed to participate in the study. Inclusion criteria for participation included the following: 1) attend church services at the selected intervention 
church at least 2 times per month, 2) not currently pregnant, 3) live within 15 driving minutes from the church, 4) not been diagnosed by a physician with heart problems, and 5) have no intention of moving from the area within the year of enrollment.

\section{Procedures}

Following institutional approval of study protocol, participants completed a 12-page questionnaire in the language of their choice (English or Spanish). The survey consisted of a 12-page questionnaire available in both English and Spanish (99\% of participants chose Spanish surveys). Bilingual research assistants administered the GPAQ and participants completed the rest of the 20-minute survey on their own, if no assistance was needed. Following completion of the survey, participants were asked to wear the accelerometer for 7 days at baseline and 6 months following baseline.

Participants were asked to wear an Actigraph accelerometer (GT1M model; Actigraph, LLC, Pensacola, Florida) for 7 consecutive days at baseline and 6 months post baseline. The Actigraph measures and records uniaxial accelerations ranging in magnitude from 0.05 to $2 \mathrm{G}$ and has shown to provide valid and reliable assessments of physical activity frequency, intensity and duration among adults in both laboratory and free living environments. ${ }^{7}$ The device was set to aggregate acceleration data based on an epoch length of 60s and was attached to an elastic waist belt and positioned above the iliac crest of the right hip, consistent with the manufacturer's recommendations. Nonwear time was defined as $60 \mathrm{~min}$ of consecutive zeros. Participants were asked to remove the device during water-based activities (eg, swimming, showering) and while sleeping. ActiGraph data were downloaded using ActiLife v4 software (Actigraph, LLC) and analyzed using MeterPlus software (Santech, LLC). Participants' whose data were not recorded for a minimum of 8 hours for 4 days were excluded. ${ }^{7}$ Outcome data were expressed as minutes per day in sedentary, light, moderate, and vigorous intensity using the cut points proposed by Freedson and colleagues. ${ }^{15}$

The Global Physical Activity Questionnaire (GPAQ) was used to assess PA. The World Health Organization (WHO) developed the GPAQ to measure the PA habits of populations using a standardized protocol. ${ }^{16}$ The GPAQ assesses 3 domains of PA and sedentary behavior: occupational (PA at work), active commuting (PA for travel), and recreational (leisure time PA). Of note, household activity was included as part of the work domain.

The GPAQ has been shown to be valid and reliable, but also adaptable to incorporate cultural and other differences. ${ }^{17}$ On a continuous scale, participants were asked to report on their physical activity during a typical week. The average minutes per week were computed based on the participant's dichotomous response (yes or no) to whether they engaged in PA and the type, intensity, duration, and frequency of PA in which they participated. A sum total was created for participants who answered yes to engaging in PA based on the following equation: minutes engaged in PA multiplied by the average number of days per week engaged in PA. A summary variable of average minutes per week of moderate-tovigorous PA (MVPA) was calculated for each domain of PA by combining the average minutes per week of both moderate and vigorous PA for each PA domain.

Participants' age, marital status, number of children, employment status, education level, nationality/family country of origin, years lived in the U.S, and income range were assessed in the 12-page survey.

Participants were asked to self-report their height $(\mathrm{cm} \rightarrow \mathrm{ft})$ and weight $(\mathrm{kg} \rightarrow \mathrm{lbs})$ and their BMI was calculated using the Quetelet Index, where BMI $=$ [body mass $(\mathrm{lbs}) \times 703] /$ 
[height (inches)]. ${ }^{18} \mathrm{~A}$ BMI of 25.0 to 29.9 was used as the cut point for overweight and a BMI above 30.0 was considered obese.

\section{Data Analysis}

Histograms and Shapiro-Wilk tests were used to evaluate normality of all variables. Nonparametric tests, Spearman's Rank Correlation Coefficient were used for all correlation analyses. The square root was taken of each variable to approximate normality. For participants with valid data at baseline and postintervention, summary variables of sedentary, light, moderate, and vigorous PA minutes per week were calculated from the accelerometer data. Summary variables of minutes per week for light, moderate, and vigorous activity during work, chores, travel and leisure time were calculated from participants' responses to the GPAQ. To estimate the association between self-reported activity on the GPAQ and data from the accelerometer, one-tailed nonparametric Spearman's correlation coefficients were used. To preserve a family-wise type I error rate of 5\%, a Bonferroni correction was applied and a significance level was determined by $P<.004$. To test the sensitivity of the GPAQ for detecting change in PA, differences in minutes per week of activity were computed as postintervention minus baseline for each participant. Spearman correlation coefficients were used to examine associations between the change scores by method of assessment All statistical analysis was performed using the PASW (Predictive Analytic Software) Version 17.

\section{Results}

Characteristics of the sample are shown in Table 1 . The majority of the participants were unemployed, married, with at least 1 child under the age of 18 living at home. Nearly onethird of participants were obese. The mean acculturation score for participants, from the Short 12 item Acculturation Scale for Hispanics ${ }^{19}$ was 11.61 (SD = 4.33; range = 7-32), indicating lower acculturation levels for most participants.

\section{GPAQ and Accelerometer Data}

Table 2 shows summary estimates of mean minutes per week of accelerometer and GPAQ data at baseline and at 6 months. Accelerometer data show a decrease from baseline to 6 months in minutes per week of sedentary activity, vigorous PA and total PA, and an increase in minutes per week of light, moderate, and MVPA. Self-reported minutes per week of occupational activity (moderate and vigorous), commuting-related PA, chores, sedentary activity, total moderate PA, and MVPA decreased from baseline to 6 months. Self-reported minutes per week of leisure time PA and total vigorous PA increased from baseline to 6 months. Relationships between self reported and accelerometer measured minutes per week of PA were examined and discussed below.

\section{Cross-Sectional Relationships Between GPAQ and Accelerometer Data}

The association between accelerometer and self-reported minutes per week of PA across domains and by intensity level are presented in Table 3 and Table 4 . At baseline, there were significant correlations between self-report minutes per week of light chores and accelerometer measured light PA $(r=.328, P<.004)$, total vigorous activity and accelerometer measured light PA $(r=.324, P<.003)$, vigorous LTPA and accelerometer measured vigorous PA $(r=.521, P<.001)$, total vigorous PA and accelerometer measured vigorous PA $(r=.423, P<.000)$, and leisure time MVPA and accelerometer measured MVPA $(r=.376, P<.002)$. There was a significant correlation between self-report minutes per week of vigorous LTPA and accelerometer measured vigorous PA 6 months postbaseline $(r=.404, P<.001)$. 


\section{Sensitivity of GPAQ for Detecting Intervention Change in PA}

To assess the sensitivity of the GPAQ for detecting change in PA, we examined the difference between self-report estimates and accelerometer estimates from baseline to post intervention. It was hypothesized that change measured by the GPAQ (baseline to post intervention) would have a strong correlation to change measured by the accelerometer, based on the hypothesis that increased participation in the intervention was positively associated with accuracy in reporting PA. The difference in minutes per week of activity (post intervention minus baseline) between self-reported minutes per week of accelerometer data were calculated and correlated across each level of PA. The GPAQ was sensitive to change in self-reported vigorous LTPA and accelerometer measured vigorous PA ( $r=.383$, $P<.003)$, and self-reported total vigorous PA and accelerometer measured vigorous PA $(r$ $=.363, P<.003$; Table 5).

\section{Impact of Intervention Attendance on Correlations Between GPAQ and Accelerometer Data}

One of the objectives of this study was to assess whether regular intervention attendance contributes to increased accuracy of self-report data as compared with data collected by accelerometers. If intervention attendance did contribute to increased accuracy of selfreported minutes per week of activity then it was hypothesized that correlations between self-report and accelerometer measured data at post intervention would be stronger for participants who regularly attended the intervention. To examine this association, participants' were stratified into 2 groups; low attendance (those who attended the intervention once a week or less) and high-attendance (those who attended once a week or more.) There were stronger correlations postintervention for the low attendance group for self-reported moderate LTPA and accelerometer measured moderate PA $(r=.161, .220)$, and self-reported and accelerometer measured sedentary behavior $(r=.219, .283)$. When examining the data of participants who regularly attended the intervention, there were stronger correlations postintervention for self-reported total vigorous PA and accelerometer measured vigorous PA $(r=.389, .419)$, self-reported vigorous LTPA and accelerometer measured vigorous PA $(r=.291, .613)$, self-reported activity for travel and accelerometer measured moderate PA $(r=-.019, .298)$, and self-reported MVPA at work and accelerometer measured MVPA $(r=-.017,-.299)$.

\section{Discussion}

This study contributes to current research by examining the validity of the GPAQ in the Latina population, a community with high rates of physical inactivity. ${ }^{1}$ In addition, this study also examined at the sensitivity of the GPAQ, which could benefit further intervention studies involving Latinas that use self-report measures. Currently there are very few studies examining self-report measures within the Latina population, by validating the effectiveness of the GPAQ in this population it could aid future research aiming to measure vigorous activity among Latinas. In addition, this study adds to the current evidence base to better understand the impact of interventions targeting Latinos, as it is important to examine the extent to which culturally appropriate measures are sensitive to intervention-changes in behavior.

This study sought to determine whether the GPAQ is a valid self-report tool for assessing PA level of Latinas by assessing the correlation between PA levels recorded by accelerometers, and PA estimates produced by the GPAQ. We found a) significant crosssectional correlations between different intensity levels across select domains, b) the GPAQ was sensitive to changes only for vigorous activity across time points, and c) those who participated in intervention activities were more accurate reporters of activity than those who participated less frequently. 
Study results suggest that the GPAQ may be a more accurate measure of vigorous PA than light and moderate intensity PA. In a review of GPAQ validity data, ${ }^{20}$ discrepancies between self-reported and objectively measured PA were greater for higher intensity PA (ie, vigorous). Other studies have also found high correlations between self-report and objectively measured vigorous PA. ${ }^{10,21}$

Data from this study reveal significant preintervention correlations between accelerometry recorded measures and self-reported measures of light chores, vigorous LTPA, total vigorous PA, and total LTPA. Postintervention, significant correlations were found for vigorous LTPA. Consistent with previous research, participants in this study may have had a difficult time estimating moderate intensity PA, which is known to be difficult both perceptually and cognitively. ${ }^{22,23}$ Our findings corroborate that of previous studies ${ }^{10,21}$ highlighting that vigorous intensity PA might be easier to recall and therefore more accurately reported than lower intensity activity.

The discrepancy between self-reported and accelerometer measured PA may be due to the inability of accelerometers to account for upper body movement, which may increase in work and chore-related PA. A significant amount of housework, such as doing laundry or washing dishes, may involve limited movement of the center of mass which is required for the accelerometer to detect activity. Similar findings have been reported in other studies. $^{20,24}$

In this study, there was a higher correspondence between self-reported PA data and the accelerometer data at baseline compared with 6 months. It may be that participants were highly motivated at the beginning of the intervention and as a result were better at recalling PA at baseline. After 6 months, the novelty of the intervention may have waned and participants could be less motivated to accurately report their PA. Furthermore, it may be that because the current intervention aimed at promoting physical activity, participants were more likely to report engaging in higher levels of PA post intervention. Thus, social desirability might have added additional measurement error ("noise") to the self-reported data post intervention, which makes it harder to detect true PA (the "signal").

Results also revealed that data from participants who regularly attended the intervention (defined as attending the intervention 1 or more times per week) had stronger correlations postintervention for total vigorous PA, vigorous LTPA, activity for travel, and total activity at work, suggesting that regular attendance in the intervention is associated with more accurate reports of PA for select domains. Consistent with previous research, higher attendance was associated with more accurate reporting. ${ }^{25,26}$ Participants who attended the intervention more regularly may have found it easier to accurately report minutes of PA or this may be a measurement artifact as a result of increased variance in vigorous PA due to the intervention.

This study has several limitations. This study involved a convenience sample of intervention volunteers which limits the generalizability of the findings; however this study's findings support the need to expand on research validating self-report measures among Latinas as there is a lack of research in current literature. Furthermore, the lack of a control group makes it difficult to untangle the impact of social desirability on volunteering for a PA intervention or on the accuracy of PA reports. Social desirability might have suppressed the association between self-reported PA and accelerometer data, making it difficult to find more sensitivity effects in the self-reported of PA. Lastly, the inability of the accelerometer to measure upper body movement may have downwardly biased the assessment of objectively assessed PA minutes. 
Identifying ways to increase the accuracy of self-report measures among Latinas is important in monitoring PA rates and trends, as well as efforts to assess intervention effectiveness. Studies would benefit from including both modality of PA assessment because each provides important and unique data. Self-reported assessments help describe the types of activity participants are engaging in (ie, occupational) whereas the accelerometer is more valid for measuring the intensity of activity. While other self-report questionnaires have been validated against the GPAQ, to our knowledge, this is the first study to assess the validity of the GPAQ among U.S. Latinas. Increasing the accuracy of self-report tools will improve the quality of the data collected and provide a more accurate measure of the association between PA and health outcomes.

\section{Acknowledgments}

This work was supported by award number T32GM084896 (PI: M. Hovell) from the National Institute of General Medical Sciences (Nancy Espinoza) and grant \#65337 from the Robert Wood Johnson Foundation and NCI R21CA122471 (Dr. Arredondo). The content is solely the responsibility of the authors and does not necessarily represent the official views of the National Institute of General Medical Sciences or the National Institutes of Health.

\section{References}

1. National Heart Lung and Blood Institute. [November 1, 2008] The heart truth for Latinas: an action plan. http://www.nhlbi.nih.gov/ health/hearttruth/material/factsheet_latina.pdf.

2. Center for Disease Control and Prevention. Health disparities experienced by Hispanics-United States. MMWR Morb Mortal Wkly Rep. 2004; 53:935-937. PubMed. [PubMed: 15483525]

3. U.S. Department of Health and Human Services. [October 26, 2009] Health, United States. 2008. http://www.cdc.gov/nchs/data/hus/hus08.pdf.

4. Pew Hispanic Center. [November 2, 2008] Factsheet: Hispanic women in the United States. 2007. http://pewhispanic.org/factsheets/fact-sheet.php?FactsheetID=42.

5. Schmidt MD, Freedson PS, Chasan-Tabar L. Estimating physical activity using the CSA accelerometer and a physical activity log. Med Sci Sports Exerc. 2003; 35:1605-1611. PubMed doi: 10.1249/01.MSS.0000084421.97661.17. [PubMed: 12972884]

6. Sloane R, Snyder DC, Demark-Wahnefried W, Lobach D, Kraus WE. Comparing the 7-day physical activity recall with a triaxial accelerometer for measuring time in exercise. Med Sci Sports Exerc. 2009; 41:1334-1340. PubMed doi:10.1249/MSS.0b013e3181984fa8. [PubMed: 19461530]

7. Trost SG, McIver KL, Pate RR. Conducting accelerometer-based activity assessments in field-based research. Med Sci Sports Exerc. 2005; 37:S531-S543. PubMed doi:10.1249/01.mss. 0000185657.86065.98. [PubMed: 16294116]

8. Martinez-Gonzalez MA, Lopez-Fontana C, Varo JJ, Sanchez-Villegas A, Martinez JA. Validation of the Spanish version of the physical activity questionnaire used in the nurses' health study and the health professionals' follow-up study. Public Health Nutr. 2005; 8:920-927. PubMed doi:10.1079/ PHN2005745. [PubMed: 16277809]

9. Hardy LL, Booth ML, Okely AD. The reliability of the Adolescent Sedentary Activity Questionnaire (ASAQ). Prev Med. 2007; 45:71-74. PubMed doi:10.1016/j.ypmed.2007.03.014. [PubMed: 17532371]

10. MacKay LM, Schofield GM, Schliuter PJ. Validation of self-report measures of physical activity: a case study using the New Zealand physical activity questionnaire. Res Q Exerc Sport. 2007; 78:189-197. PubMed doi:10.5641/193250307X13082490460904. [PubMed: 17679492]

11. Sallis JF, Saelens BE. Assessment of physical activity by self-report: status, limitations, and future directions. Res Q Exerc Sport. 2002; 71:1-14. PubMed.

12. Aadahl M, Jorgensen T. Validation of a new self-report instrument for measuring physical activity. Med Sci Sports Exerc. 2003; 35:1196-1202. PubMed doi:10.1249/01.MSS.0000074446.02192.14. [PubMed: 12840642] 
13. Reis JP, Dubose KD, Ainsworth BE, Macera CA, Yore MM. Reliability and validity of the Occupational Physical Activity Questionnaire. Med Sci Sports Exerc. 2005; 37:2075-2083. PubMed doi:10.1249/01.mss.0000179103.20821.00. [PubMed: 16331132]

14. U.S Census Bureau. [November 13, 2009] State and county quick facts. http:// quickfacts.census.gov/qfd/states/06/0613392.html.

15. Freedson PS, Melanson E, Sirard J. Calibration of the Computer Science Applications, Inc. accelerometer. Med Sci Sports Exerc. 1998; 30:777-781. PubMed doi: 10.1097/00005768-199805000-00021. [PubMed: 9588623]

16. Bull FC, Maslin TS, Armstrong T. Global Physical Activity Questionnaire (GPAQ): nine country reliability and validity study. J Phys Act Health. 2009; 6:790-804. PubMed. [PubMed: 20101923]

17. Wolin KY, Heil DP, Askew S, Matthews CE, Bennett GG. Validation of the International Physical Activity Questionnaire-Short among Blacks. J Phys Act Health. 2008; 5:746-760. PubMed. [PubMed: 18820348]

18. Hedley AA, Ogden CL, Johnson CL, Carroll MD, Curtin LR, Flegal KM. Prevalence of overweight and obesity among US children, adolescents, and adults, 1999-2002. JAMA. 2004; 291:2847-2850. PubMed doi:10.1001/jama.291.23.2847. [PubMed: 15199035]

19. Marín G, Sabogal F, VanOss Marín B, Otero-Sabogal F, Pérez-Stable EJ. Development of a short acculturation scale for Hispanics. Hisp J Behav Sci. 1987; 9:183-205. doi: 10.1177/07399863870092005.

20. Paternoster R, Brame R, Mazerolle P, Piquero A. Using the correct statistical test for the equality of regression coefficients. Criminology. 1998; 36:859-866. doi:10.1111/j. 1745-9125.1998.tb01268.x.

21. Prince SA, Adamo KB, Hamel ME, Hardt J, Connor Gorber S, Tremblay M. A comparison of direct versus self-report measures for assessing physical activity in adults: a systematic review. Int J Behav Nutr Phys Act. 2008; 5:56-80. PubMed doi:10.1186/1479-5868-5-56. [PubMed: 18990237]

22. Kurtze N, Rangul V, Hustvedt B. Reliability and validity of the international physical activity questionnaire in the Nord-Trøndelag health study (HUNT) population of men. BMC Med Res Methodol. 2008; 8:63-71. PubMed doi:10.1186/1471-2288-8-63 23. [PubMed: 18844976]

23. Altschuler A, Picchi T, Nelson M, Rogers JD, Hart J, Sternfeld B. Physical activity questionnaire comprehension: lessons from cognitive interviews. Med Sci Sports Exerc. 2009; 4:336-343. PubMed. [PubMed: 19127192]

24. Wilcox S, Irwin ML, Addy C, et al. Agreement between participant-rated and compendium-coded intensity of daily activities in a triethnic sample of women ages 40 years and older. Ann Behav Med. 2001; 23:253-262. PubMed doi:10.1207/S15324796ABM2304_4. [PubMed: 11761342]

25. Hawkins MS, Storti KL, Richardson CR, et al. Objectively measured physical activity of USA adults by sex, age, and racial/ethnic groups: a cross-sectional study. Int J Behav Nutr Phys Act. 2009; 6:31-37. PubMed doi:10.1186/1479-5868-6-31. [PubMed: 19493347]

26. Stevens VJ, Obarzanek E, Cook NR, et al. Long-term weight loss and changes in blood pressure: results of the trials of hypertension prevention, phase II. Ann Intern Med. 2001; 134:1-11. PubMed. [PubMed: 11187414] 
Table 1

Characteristics of Sample

\begin{tabular}{llc}
\hline & & $\%$ \\
\hline Ethnicity & Latinas $(\mathrm{N}=71)$ & 100 \\
Age & $<43$ years & 62.9 \\
$\#$ of children under 18 in home & 0 children & 14.5 \\
BMI & BMI <30 & 37.7 \\
Education level & High school or less & 65.7 \\
Marital status & Married or living as married & 82.9 \\
Income level & $<$ \$2000 per month & 49.3 \\
Place of birth & Mexico or outside the US & 95.7 \\
Lived in US & 10 years or less & 42.0 \\
Lived at present location & 3 years or less & 48.6 \\
Housing status & Rent apartment or house & 51.4 \\
Current employment & Employed & 47.2 \\
Able to drive & No & 17.1 \\
\hline
\end{tabular}


Table 2

Mean Minutes per Week of Accelerometer and Self-Reported PA

\begin{tabular}{|c|c|c|c|}
\hline & Baseline & 6 months & Mean change (\%) \\
\hline Accelerometer (min/week) & Mean (SD) & Mean (SD) & Baseline at 6 months \\
\hline Sedentary & $3365.79(530.43)$ & $3221.94(560.63)$ & $143.85(-4.3 \%)$ \\
\hline Light PA & $2347.75(462.16)$ & $2495.16(530.48)$ & $147.41(+6.3 \%)$ \\
\hline Mod PA & $148.77(93.61)$ & $161.14(108.35)$ & $12.37(+8.31 \%)$ \\
\hline Vig PA & $6.41(19.09)$ & $5.92(16.25)$ & $.49(-7.6 \%)$ \\
\hline Mod-to-Vig PA & $155.18(99.03)$ & $167.06(114.53)$ & $11.88(+7.7 \%)$ \\
\hline Total PA & $2502.92(494.25)$ & $2501.08(531.03)$ & $1.84(0)$ \\
\hline \multicolumn{4}{|l|}{ GPAQ (min/week) } \\
\hline Vig PA at work & $203.33(718.43)$ & $159.15(517.84)$ & $44.18(-21.7 \%)$ \\
\hline Moderate PA at work & $346.29(732.14)$ & $231.31(557.77)$ & $114.98(-33.2 \%)$ \\
\hline Total PA at work & $554.64(1279.88)$ & $390.46(943.52)$ & $164.18(-29.6 \%)$ \\
\hline PA for travel & $242.93(464.27)$ & $185.69(380.65)$ & $57.24(-23.6 \%)$ \\
\hline Vig LTPA & $127.61(194.77)$ & $153.77(192.68)$ & $26.16(+20.5 \%)$ \\
\hline Mod LTPA & $115.64(174.61)$ & $149.08(120.60)$ & $33.44(+28.9 \%)$ \\
\hline Total LTPA & 244.93 (325.99) & 302.85 (239.06) & $57.92(+23.64 \%)$ \\
\hline Sedentary behavior & $1130.14(915.49)$ & $1052.69(981.06)$ & $77.45(-6.8 \%)$ \\
\hline Light chores & $1680.86(1396.33)$ & $1099.85(778.11)$ & $581.01(-34.6 \%)$ \\
\hline Mod-to-Vig chores & $743.07(971.80)$ & $478.66(631.24)$ & $264.41(-35.6 \%)$ \\
\hline Total moderate PA & $704.86(917.75)$ & $566.08(700.31)$ & $138.78(-19.7 \%)$ \\
\hline Total vig PA & $329.19(748.51)$ & $312.92(541.60)$ & $16.87(-5.1 \%)$ \\
\hline Total PA & $1020.37(1407.56)$ & 879 (1051.89) & $141.37(-13.9 \%)$ \\
\hline
\end{tabular}

Abbreviations: Vig, vigorous; Mod, moderate; LTPA, leisure-time physical activity; PA, physical activity, GPAQ, Global Physical Activity Questionnaire. 


\section{Table 3}

Spearman's Correlation Coefficients of Accelerometer and Self-Report Mean Minutes per Week of PA at Baseline

\begin{tabular}{|c|c|c|c|c|c|c|}
\hline \multirow[b]{3}{*}{ GPAQ } & \multicolumn{6}{|c|}{ Accelerometer } \\
\hline & Sedentary & $\underline{\text { Light PA }}$ & $\underline{\operatorname{Mod} P A}$ & Vig PA & Mod-to-vig PA & Total PA \\
\hline & Baseline & Baseline & Baseline & Baseline & Baseline & Baseline \\
\hline Sedentary & 0.28 & $-0.34^{* *}$ & 0.02 & -0.1 & 0.03 & $0.30^{* *}$ \\
\hline Moderate PA at work & 0.09 & -0.03 & -0.15 & -0.05 & -0.09 & -0.03 \\
\hline Moderate PA for travel & 0.11 & 0.03 & 0.24 & 0.08 & $0.23^{*}$ & 0.08 \\
\hline Moderate LTPA & -0.06 & $0.26^{*}$ & 0.2 & $0.44^{* * *}$ & $0.27 *$ & $0.28^{* *}$ \\
\hline Total moderate PA & -0.02 & 0.17 & 0.04 & 0.16 & 0.09 & 0.18 \\
\hline Vig PA at work & 0.04 & 0.1 & -0.24 & 0.03 & -0.17 & 0.07 \\
\hline Vig LTPA & -0.12 & 0.29 & 0.25 & $0.52^{* * *}$ & $0.32^{* *}$ & 0.28 \\
\hline Total Vig PA & -0.12 & $0.32^{* *}$ & 0.07 & $0.42^{* *}$ & 0.15 & $0.34^{* *}$ \\
\hline Total PA at work & 0.12 & -0.07 & -0.23 & -0.07 & -0.17 & -0.07 \\
\hline LTPA & -0.12 & 0.28 & $0.30^{* *}$ & $0.53^{* *}$ & $0.38^{* *}$ & $0.33^{* *}$ \\
\hline Total PA & -0.07 & 0.2 & 0.08 & 0.28 & 0.14 & 0.22 \\
\hline Light chores & -0.07 & $0.33^{* *}$ & -0.06 & 0.06 & -0.05 & $0.28^{* *}$ \\
\hline Mod-to-Vig chores & -0.04 & 0.24 & -0.02 & 0.15 & 0.02 & 0.22 \\
\hline
\end{tabular}

Abbreviations: Vig, vigorous; Mod, moderate; LTPA, leisure-time physical activity; PA, physical activity, GPAQ, Global Physical Activity Questionnaire.

*** Significant at the .004 level 
Table 4

Spearman's Correlation Coefficients of Accelerometer and Self-Report Mean Minutes per Week of Physical Activity at 6 Months

\begin{tabular}{|c|c|c|c|c|c|c|}
\hline \multirow[b]{3}{*}{ GPAQ } & \multicolumn{6}{|c|}{ Accelerometer } \\
\hline & Sedentary & Light PA & Mod PA & Vig PA & Mod-to-vig PA & Total PA \\
\hline & 6 months & 6 months & 6 months & 6 months & 6 months & 6 months \\
\hline Sedentary & 0.25 & $-0.41^{* *}$ & -0.07 & -0.19 & -0.06 & 0.00 \\
\hline Moderate PA at work & 0.13 & 0.03 & -0.1 & -0.1 & -0.12 & 0.03 \\
\hline Moderate PA for travel & -0.06 & 0.05 & 0.04 & 0.1 & 0.04 & 0.06 \\
\hline Moderate LTPA & -0.05 & -0.05 & 0.21 & 0.01 & 0.22 & 0.04 \\
\hline Total moderate PA & 0.04 & 0.01 & -0.02 & -0.03 & -0.02 & 0.03 \\
\hline Vig PA at work & 0.13 & 0.03 & -0.24 & -0.19 & -0.25 & -0.03 \\
\hline Vig LTPA & -0.08 & -0.02 & 0.19 & $0.40^{* *}$ & 0.21 & 0.07 \\
\hline Total Vig PA & -0.02 & 0.04 & 0.05 & 0.24 & 0.24 & 0.04 \\
\hline Total PA at work & 0.11 & 0.06 & -0.2 & -0.15 & -0.21 & 0.04 \\
\hline LTPA & -0.09 & -0.08 & 0.26 & $0.29^{* *}$ & 0.27 & 0.03 \\
\hline Total PA & 0.00 & 0.03 & -0.06 & 0.05 & -0.06 & 0.03 \\
\hline Light chores & -0.2 & 0.15 & 0.17 & 0.16 & 0.18 & 0.19 \\
\hline Mod-to-vig chores & -0.11 & -0.12 & 0.02 & 0.05 & 0.02 & -0.06 \\
\hline
\end{tabular}

Abbreviations: Vig, vigorous; Mod, moderate; LTPA, leisure-time physical activity; PA, physical activity, GPAQ, Global Physical Activity Questionnaire.

*** Significant at the .004 level. 
Table 5

Spearman Correlation Coefficients of Sensitivity of GPAQ to Assess Differences (post- pre) in Reported PA

\begin{tabular}{lcccccc}
\hline & \multicolumn{5}{c}{ Accelerometer } \\
\cline { 2 - 6 } GPAQ & Sedentary & Light PA & Mod PA & Vig PA & Mod-to-vig PA & Total PA \\
\hline Sedentary & -0.01 & -0.22 & 0.00 & 0.01 & -0.01 & -0.16 \\
Moderate PA at work & 0.05 & -0.14 & 0.17 & 0.13 & 0.20 & -0.10 \\
Moderate PA for travel & -0.08 & 0.17 & -0.08 & -0.03 & -0.07 & 0.17 \\
Moderate LTPA & -0.10 & 0.13 & 0.13 & 0.13 & 0.17 & 0.17 \\
Total moderate PA & 0.01 & 0.11 & 0.11 & 0.06 & 0.15 & 0.14 \\
Vig PA at work & -0.01 & -0.09 & -0.07 & 0.11 & -0.04 & -0.10 \\
Vig LTPA & -0.03 & 0.03 & 0.04 & $0.38 *$ & 0.08 & 0.08 \\
Total vig PA & -0.01 & -0.05 & 0.08 & $0.36 *$ & 0.12 & 0.00 \\
Mod-to-vig PA at work & 0.03 & -0.15 & 0.00 & 0.15 & 0.04 & -0.15 \\
Mod-to-vig LTPA & -0.14 & 0.07 & 0.12 & 0.29 & 0.16 & 0.13 \\
Mod-to-vig PA & -0.01 & 0.00 & 0.09 & 0.23 & 0.14 & 0.05 \\
Light chores & 0.31 & 0.10 & -0.24 & 0.15 & -0.21 & 0.01 \\
Mod-to-vig chores & 0.09 & -0.10 & -0.03 & 0.01 & -0.03 & -0.13 \\
\hline ** & & & & & & \\
Significant at the .004 level. & & & & & \\
\end{tabular}

